CERN-TH.7556/95

\title{
Higgs Boson Production in $e^{+} e^{-} \rightarrow \mu^{+} \mu^{-} b \bar{b}$
}

\author{
Guido MONTAGNA ${ }^{a}$, Oreste NICROSINI ${ }^{b}$ 田 and Fulvio PICCININI ${ }^{c}$ \\ ${ }^{a}$ INFN, Sezione di Pavia, Italy \\ ${ }^{b}$ CERN, TH Division, Geneva, Switzerland \\ ${ }^{c}$ Dipartimento di Fisica Nucleare e Teorica, Università di Pavia, and \\ INFN, Sezione di Pavia, Italy
}

\begin{abstract}
The production of the Standard Model Higgs boson in the fourfermion reaction $e^{+} e^{-} \rightarrow \mu^{+} \mu^{-} b \bar{b}$ is studied. The complete tree-level matrix element, including signal and backgrounds in the standard electroweak theory, is computed and initial state radiation is taken into account in the leading-log approximation. A Monte Carlo event generator has been built and numerical results for some distributions of experimental interest for the search of the Higgs particle at future electron-positron colliders are shown, compared with those existing in the literature and commented.
\end{abstract}

\section{Submitted to Physics Letters B}

E-mail:

montagna@pv.infn.it

nicrosini@vxcern.cern.ch

piccinini@pv.infn.it

CERN-TH.7556/95

January 1995

\footnotetext{
${ }^{1}$ On leave from INFN, Sezione di Pavia, Italy.
} 
After the experimental confirmations of the standard theory of the electroweak interaction provided by the precision tests at LEP/SLC [1] and by the evidence of top quark production at the TEVATRON [2], the search for the Standard Model Higgs boson becomes a primary task of the experiments planned at future electron-positron and hadron colliders. For the case of a light or intermediate Higgs particle $\left(M_{H} \leq 2 M_{W}\right)$, the Bjorken bremsstrahlung process $e^{+} e^{-} \rightarrow H Z$ is known to be the main production mechanism at $e^{+} e^{-}$colliders up to $0.5 \mathrm{TeV}$ [3]. Detailed studies about the production of the Higgs boson in $e^{+} e^{-}$collisions are given in Refs. [4, 5]. From these investigations it emerges that the specific four-fermion reaction

$$
e^{+} e^{-} \rightarrow H Z \rightarrow \mu^{+} \mu^{-} b \bar{b}
$$

provides the cleanest event signature coupled with "small" backgrounds.

A complete tree-level calculation of the four-fermion process (11), including all possible diagrams and their interferences, has been performed in two recent papers [6, 7]. On the one hand, in Ref. [6] a Monte Carlo approach has been adopted but the effect of the initial state QED corrections, large and hence necessary for the data analysis, has been neglected; on the other hand, initial state radiation has been included in [7], but according to a semi-analytical procedure which is limited from the point of view of event simulation purposes. The aim of this letter is to present a Monte Carlo event generator for the process $e^{+} e^{-} \rightarrow \mu^{+} \mu^{-} b \bar{b}$ based on the calculation of the full set of diagrams contributing in the standard electroweak theory to the assigned four-fermion final state and on the inclusion of initial state QED corrections in the leading-log approximation. We will only sketch the basic theoretical ingredients, putting more emphasis on the phenomenological aspects of the study and on the potentials of a "specialized" event generator for future searches of the Higgs boson at LEP2 and NLC energies.

The kinematics of the $2 \rightarrow 4$ process at the Born level has been treated according to the general recipe for a four-fermion final state described in [\$] Essentially, seven independent dimensionless quantities are introduced and chosen, together with a trivial overall azimuthal angle, as independent variables from which the full four-momenta of the outgoing particles are reconstructed in the laboratory frame by explicit solution of the kinematics.

The inclusion of initial state radiation is simply achieved by dressing the incoming leptons with QED structure functions [9]. They allow to include, 
by virtue of factorization theorems, the long-distance universal contributions in soft and/or collinear approximation.

Taking into account the initial state radiation, the total cross section can be expressed as a nine-fold convolution of the form

$$
\sigma(s)=\sum_{i} \int d x_{1} d x_{2} D\left(x_{1}, s\right) D\left(x_{2}, s\right) d[P S] \frac{d \sigma}{d[P S]} \frac{w_{i}}{W}
$$

where $d[P S]$ denotes the volume element in the 7-dimensional phase space connected to the independent variables of the hard scattering reaction in the c.m. frame. The scale of the hard scattering cross section is the reduced c.m. energy $\hat{s}=x_{1} x_{2} s$ which is the main effect of the initial state radiation together with the boost of the c.m. frame with respect to the laboratory one, which is also taken into account by properly rescaling the independent variables of the hard kernel cross section in the c.m. frame.

In the kernel cross section the complete set of tree-level diagrams contributing in the Standard Model to $e^{+} e^{-} \rightarrow \mu^{+} \mu^{-} b \bar{b}$ is included. Twenty-five diagrams have to be computed and they can be classified as follows:

- the signal diagram $e^{+} e^{-} \rightarrow H Z$ followed by the decays $H \rightarrow b \bar{b}$ and $Z \rightarrow \mu^{+} \mu^{-}$

- the eight $t$-channel diagrams relative to $Z Z, \gamma \gamma, Z \gamma$ exchange;

- the sixteen $s$-channel diagrams corresponding to the bremsstrahlung of a neutral vector boson from the fermionic final states (radiative processes).

Each amplitude has been computed by means of SCHOONSCHIP [10] within the helicity amplitude formalism [11] following the general strategy described in 8]. This allows to express the kernel cross sections essentially as the sum of products of resonating propagators with functions of the dimensionless variables originating from the calculation of the traces of the $\gamma$ matrices' strings. Mass corrections have been neglected in the kinematics and in the calculation of the amplitudes. These contributions introduce terms of the order of $m_{b}^{2} / s$ which at LEP2 energies amount to about $0.1 \%$ and hence they can be neglected as long as the ratio signal / background is larger than some per cent. 
To take care of the peaking behaviour of the integrand the Monte Carlo event generation is treated according to the importance sampling technique 12. The latter is applied, by means of proper changes of variables, to the exponentiated infrared sensitive part of the structure functions, to the different propagators' "singularities" (particularly to the Higgs propagator which resembles for its very narrow width a delta-like function) and finally to the jacobian peaks appearing in the phase space factor. In particular, the weights $w_{i}$ and $W=\sum_{i} w_{i}$ are introduced to obtain a proper sampling of the double propagator "singularities" of the signal $(Z H)$ and the backgrounds $(Z Z, Z \gamma$ and $\gamma \gamma$ in all possible combinations).

Let us show and comment some results obtained with our event generator based on the random number generator RANLUX [13]. The input parameters used are: $M_{Z}=91.1887 \mathrm{GeV}, \Gamma_{Z}=2.4974 \mathrm{GeV}, M_{W}=80.22 \mathrm{GeV}, m_{b}=$ $4.7 \mathrm{GeV}$ [14]. For the Higgs boson width the tree-level expression is adopted.

In Fig. 1a the total cross section in Born approximation is compared with the initial state QED-corrected one as a function of the c.m. energy and for three different values of the Higgs mass. The dashed, dash-dotted and closely dotted lines represent the lowest-order results for $M_{H}=65,100,140 \mathrm{GeV}$, respectively; the three solid lines refer to the above Higgs mass values and include the effect of the leading-log initial state QED corrections. As a reference curve, the QED corrected total cross section obtained by excluding the Higgs boson contribution is also shown (sparsely dotted line). Cuts on the invariant masses of the $\mu^{+} \mu^{-}$and $b \bar{b}$ pairs of $M_{\mu^{+} \mu^{-}} \geq 12 \mathrm{GeV}$ and $M_{b \bar{b}} \geq 12 \mathrm{GeV}$ (typically used in other numerical studies [6, 7]) are applied in order to reduce a large fraction of the $\gamma \gamma$ background. The values obtained for the cross sections with our event generator have been compared in detail (i.e. for different Higgs masses and c.m. energies) with the independent results of Refs. [6, 7] and found to be in good agreement. It should be noted that in the LEP2 energy range the size of the total cross section of the $\mu^{+} \mu^{-} b \bar{b}$ reaction can give by itself evidence of Higgs boson production in the "light mass" interval $65 \mathrm{GeV} \leq M_{H} \leq 100 \mathrm{GeV}$ (see also Fig. 1b), whereas for higher Higgs mass values a c.m. energy larger than $200 \mathrm{GeV}$ is necessarily required to distinguish the signal from the background. This feature, already pointed out in Ref. [6] for the lowest-order cross section, is not largely affected by initial state bremsstrahlung which essentially lowers the peak cross section of about $10 \%$ and originates a moderate radiative tail at high energies $(\sqrt{s} \geq 300 \mathrm{GeV})$, as generally occurs for other "double 
resonant" processes $(W W, Z Z)$ above threshold. Moreover, the two-peak shape for $M_{H}=65 \mathrm{GeV}$ and $M_{H}=140 \mathrm{GeV}$ is made more flat by effect of the initial state radiation, which however does not cancel the two-bump structure. Their appearing is given, with very good approximation, by the incoherent sum of the signal and backgrounds diagrams. In agreement with Ref. [6], we checked that the interference terms between diagrams with different intermediate bosons and also $s$-channel radiative processes give a small contribution.

Figure $1 \mathrm{~b}$ shows the ratio $\sigma_{s} / \sigma_{f}$ as a function of the Higgs mass and for three different energies of interest at LEP2. $\sigma_{s}$ denotes the signal cross section and $\sigma_{f}$ stands for the full four-fermion cross section including signal and backgrounds. The effect of the initial state radiation is included in this plot. As already singled out by Fig. 1a, for the case of a light Higgs mass ranging between, say $65 \mathrm{GeV}$ and $80 \mathrm{GeV}$, the ratio $\sigma_{s} / \sigma_{f}$ turns out to be more favourable for "low" centre of mass energies; on the contrary "high" centre of mass energies maintain the ratio more flat and sensitive to a Higgs mass up to, say, 100-110 GeV.

In Figs. 2-5 we show some realistic distributions of experimental interest for the Higgs search at LEP2. These results have been obtained processing the $n$-tuple created by the event generator at $\sqrt{s}=205 \mathrm{GeV}$. The full set of tree-level diagrams and the contribution of the initial state radiation have been taken into account in the simulation. A sample of $10^{4}$ four-fermion events has been generated in order to study with small statistical error the behaviour of the distributions when varying the Higgs mass. Although different cross sections correspond to different Higgs mass values, the same number of events has been considered in order to study the pattern of evolution of the shapes while varying the Higgs mass. The $b \bar{b}$ invariant mass distribution (Fig. 2), the $b$-quark scattering angle distribution (Fig. 3) and the $b \bar{b}$ relative angle distribution (Fig. 4) turn out to be useful observables to detect a Higgs signal and, if found, extract information on the quantum numbers assignment. From Fig. 2 it can be seen that the $b \bar{b}$ system invariant mass distribution exhibits two clear spikes positioned at $M_{H}$ and $M_{Z}$, as long as $M_{H}<$ say $110 \mathrm{GeV}$, the spike at $M_{H}$ resembling a delta-like function. The rise at low invariant masses is due to $\gamma \rightarrow b \bar{b}$ in $\gamma \gamma$ and $\gamma Z$ diagrams. From Fig. 3 it can be seen that the $b$-quark angular distribution shows, for the case of a light mass Higgs where the signal dominates the $\mu^{+} \mu^{-} b \bar{b}$ cross section, a clearly isotropic spin-zero behaviour gradually disappearing as increasing the 
Higgs mass. The $b \bar{b}$ relative angle distribution represented in Fig. 4 shows a two-peak structure. The peak positioned at lower $\cos \vartheta_{b \bar{b}}$ reflects the presence of $Z Z$ diagrams, while the (much smaller) one at $\cos \vartheta_{b \bar{b}} \approx 1$ comes from $\gamma \rightarrow b \bar{b}$ in the $\gamma \gamma$ and $\gamma Z$ contributions. The peak at intermediate $\cos \vartheta_{b \bar{b}}$, well visible in the case of a light mass Higgs, corresponds to the contribution of the signal diagram. The different position of the Higgs peak in the twobump shape is a consequence of the different Lorentz boost acting on the Higgs boson depending on its mass value. The residual bump at intermediate $\cos \vartheta_{b \bar{b}}$ for $M_{H}=140 \mathrm{GeV}$ comes from $Z \rightarrow b \bar{b}$ in the $\gamma Z$ contributions. Figure 5 shows two typical photon energy distributions (for $M_{H}=65 \mathrm{GeV}$ and $140 \mathrm{GeV}$, respectively) where the peak at very low energies is due to dominating soft multiphoton emission.

To summarize, we presented the results of a Monte Carlo event generator for the four-fermion reaction $e^{+} e^{-} \rightarrow \mu^{+} \mu^{-} b \bar{b}$, including all the Standard Model diagrams and the effect of initial state radiation in the leading-log approximation.

\section{References}

[1] See for instance:

D. Schaile, Forsch. f. Phys. 42 (1994) 429;

G. Altarelli, Electroweak Precision Tests: a Status Report, CERN Preprint CERN-TH. 7464/94.

[2] CDF Collaboration, F. Abe et al., Phys. Rev. Lett. 73 (1994) 225; Phys. Rev. D50 (1994) 2966.

[3] For a recent review of the Higgs phenomenology at future colliders see for instance: A. Djouadi, Report no. UdeM-GPP-TH. 94-01.

[4] V. Barger et al., Phys. Rev. D49 (1994) 79 and Proceedings of the Workshop - Munich, Annecy, Hamburg, DESY Report 93-123C, p. 5, P. M. Zerwas Ed.

[5] C.-M. J. Chen, Jiunn-Wei Chen and W.-Y. P. Hwang, Phys. Rev. D50 (1994) 4485. 
[6] E. Boos, M. Sachwitz, H. J. Schreiber and S. Shichanin, Z. Phys. C61 (1994) 675.

[7] D. Bardin, A. Leike and T. Riemann, "Semi-Analytical Approach to Higgs Production at LEP 2", Preprint DESY 94-097, CERNTH. 7305/94, LMU 08/94.

[8] G. Montagna, O. Nicrosini, G. Passarino and F. Piccinini, "Semianalytical and Monte Carlo Results for the Production of Four Fermions in $e^{+} e^{-}$Collisions", CERN Preprint CERN-TH.7497/94, to appear in Phys. Lett. B.

[9] E. A. Kuraev and V. S. Fadin, Sov. J. Nucl. Phys. 41 (1985) 466;

G. Altarelli and G. Martinelli, in Physics at LEP, CERN Report 86-02, J. Ellis and R. Peccei eds. (CERN, Geneva 1986)

O. Nicrosini and L. Trentadue, Phys. Lett. B196 (1987) 551; Z. Phys. C39 (1988) 479. For a review see also

O. Nicrosini and L. Trentadue, in Radiative Corrections for $e^{+} e^{-}$Collisions, J. H. Kühn ed. (Springer, Berlin, 1989), p. 25; in QED Structure Functions, G. Bonvicini ed., AIP Conf. Proc. No. 201 (AIP, New York, 1990), p. 12.

[10] SCHOONSCHIP, a program for symbol handling by M. Veltman, see H. Strubbe, Comput. Phys. Commun. 8 (1974) 1.

[11] G. Passarino, Nucl. Phys. B237 (1984) 249.

[12] F. James, Rep. Prog. Phys. 34 (1980) 1145.

[13] F. James, Comput. Phys. Commun. 79 (1994) 111.

[14] Review of Particles Properties, Phys. Rev. D50 (1994) 1173. 


\section{Figure Captions}

Figure 1. (a) The total cross section of $e^{+} e^{-} \rightarrow \mu^{+} \mu^{-} b \bar{b}$ without (dashed, dash-dotted and closely dotted line) and with (solid lines) initial state leading$\log$ QED corrections as a function of the c.m. energy and for different Higgs masses. The sparsely dotted line is the QED corrected total cross section obtained excluding the Higgs signal. (b) The ratio $\sigma_{s} / \sigma_{f}$ as a function of the Higgs mass in the energy range of LEP2. $\sigma_{s}$ is the Higgs signal cross section, $\sigma_{f}$ the full cross section including signal and backgrounds. QED corrections are included.

Figure 2. The $b \bar{b}$ invariant mass distribution for four different Higgs masses at $\sqrt{s}=205 \mathrm{GeV}$ (log. scale). Initial state radiation is included.

Figure 3. The $b$-quark scattering angle distribution for four different Higgs masses at $\sqrt{s}=205 \mathrm{GeV}$. Initial state radiation is included.

Figure 4 . The $b \bar{b}$ relative angle distribution for four different Higgs masses at $\sqrt{s}=205 \mathrm{GeV}$. Initial state radiation is included.

Figure 5. The photon energy distribution for two Higgs mass values at $\sqrt{s}=205 \mathrm{GeV}$ (log. scale). 
This figure "fig1-1.png" is available in "png" format from: http://arxiv.org/ps/hep-ph/9501267v1 
This figure "fig1-2.png" is available in "png" format from: http://arxiv.org/ps/hep-ph/9501267v1 
This figure "fig1-3.png" is available in "png" format from: http://arxiv.org/ps/hep-ph/9501267v1 
This figure "fig1-4.png" is available in "png" format from: http://arxiv.org/ps/hep-ph/9501267v1 
This figure "fig1-5.png" is available in "png" format from: http://arxiv.org/ps/hep-ph/9501267v1 